\title{
EDITORIAL \\ Double TALEN-edited T-cells kick B-ALL into touch
}

Gene Therapy (2017) 24, 122; doi:10.1038/gt.2017.17

CAR19 T cells can be highly effective against refractory-B-cell acute lymphoblastic leukaemia (B-ALL), but a significant limitation is the need to engineer cells for every individual patient. If engineered allogenic cells are used, there is a significant risk of host-mediated rejection of such cells and/or graft versus host disease (GvHD). In a recent study, Qasim et al. ${ }^{1}$ reported a significant step towards so-called universal CAR19 T cells (UCART19) in two paediatric patients with refractory-relapsed B-ALL using TALEN-edited allogeneic CAR19 T cells. The TALENs were used to disrupt the TRAC gene encoding the TCR alpha subunit to prevent rejection of the cells and GvHD. The gene for CD52 was also disrupted with TALENs so that the incoming CAR19 $T$ cells would not be depleted by any residual alemtuzumab, an anti-CD52 antibody used to lymphodeplete B-ALL patients prior to T-cell infusion. A third modification was expression of a 'sort/ suicide' gene, $R Q R 8$, to allow enrichment via a CD34 epitope during cell preparation, and a CD20 epitope to give the option for immunodepletion with rituximab in case of an adverse reaction. The overall clinical outcome was successful with both patients showing molecular remission within 1 month, which enabled bridging of both individuals to successful allogeneic stem cell transplants. While this approach is a positive step in the direction of universal CAR cells, it highlights the challenges and complexities of gene engineering, bordering on the field of synthetic biology.

\section{AUTHOR BIOGRAPHY}

PTH has directed a gene editing research group at University College Cork in Ireland since 2005 using ZFNs, TALENs and CRISPR to study a range of genetic diseases including cystic fibrosis and cystinosis, and skin disorders such as atopic dermatitis. He is also a Principal Investigator in the Cork SynBioCentre. His scientific journey began at his home university in Liverpool, before a long and winding journey to Cork which encompassed Glasgow (three times), Cambridge, Vienna and Edinburgh. With a background in virology, virus vectors, immunoreceptors and gene editing, he is delighted to have the opportunity to serve as Associate Editor (Europe) of Gene Therapy and looks forward to working with Rafael and the rest of the team during a very exciting era of advanced gene and cell therapies.

\section{CONFLICT OF INTEREST}

The author declares no conflict of interest.

PT Harrison

Department of Physiology, BioSciences Institute, University College Cork, Cork, Ireland E-mail: p.harrison@ucc.ie

\section{REFERENCES}

1 Qasim W et al. Molecular remission of infant B-All after infusion of universal TALEN gene-edited CAR T cells. Sci. Transl. Med 2017; 9: pii. 